\title{
The effectiveness of outpatient Thermachoice endometrial balloon ablation: a long-term 11-year outcome study
}

\author{
Vinod Kumar • Janesh Kumar Gupta
}

Received: 29 June 2013 / Accepted: 6 August 2013 /Published online: 21 August 2013

(C) Springer-Verlag Berlin Heidelberg 2013

\begin{abstract}
The aim of this study was to evaluate the long-term treatment outcomes of outpatient Thermachoice balloon endometrial ablation under a direct local anaesthetic cervical block (LA-Thermachoice). This paper is a prospective cohort study and a postal questionnaire survey in a UK teaching hospital with the participation of 253 women with heavy menstrual bleeding (HMB) undergoing LA-Thermachoice over an 11-year period between 2001 and 2011. Treatment success was measured by postoperative bleeding patterns, improvement in dysmenorrhoea, patient satisfaction and post-procedure hysterectomy rates. LA-Thermachoice was completed in $98 \%$ of women. The survey response rate was $78 \%$. The median follow-up interval was 71 months (SD 42). Seventy-nine percent of the responders reported significant improvement in $\mathrm{HMB}$ with $40 \%$ amenorrhoea rate and $86 \%$ improvement in dysmenorrhoea. Eighty-one percent felt that the benefit of procedure was maintained over a long period of time. Eighty-six percent of women were satisfied with the outcome of their procedure. The case notes of nonresponders were examined to check if any had undergone hysterectomy since the LA-Thermachoice procedure. In total, only $16 \%$ of women had a hysterectomy. This study represents the largest published series of local anaesthetic thermal endometrial ablation, reporting clinical outcomes with 11 years (median 71 months) of post-treatment follow-up. It demonstrates that the patient satisfaction with LA-Thermachoice is high, and is maintained over a long period of time after the procedure.
\end{abstract}

\footnotetext{
V. Kumar

Community Sexual and Reproductive Healthcare, University

Hospitals Birmingham NHS Foundation Trust, Birmingham, UK

J. K. Gupta $(\bowtie)$

Clinical and Experimental Medicine, University of Birmingham, Birmingham Women's NHS Foundation Trust, Edgbaston,

B15 2TG Birmingham, UK

e-mail: j.k.gupta@bham.ac.uk
}

Keywords Thermachoice endometrial ablation · Surgical management $\cdot$ Heavy menstrual bleeding · Questionnaire . Patient satisfaction

\section{Background}

Heavy menstrual bleeding (HMB) as a symptom is a very common gynaecological problem affecting $20 \%$ of women of reproductive age $[1,2]$. It can be managed medically with drugs such as tranexamic acid, mefenamic acid, oral contraceptive pills, or the levonorgestrel intrauterine system (LNGIUS, Mirena $\left.{ }^{\mathrm{TM}}\right)$ [3] or surgically with procedures such as endometrial ablation or hysterectomy.

While hysterectomy still remains the definitive surgical treatment for HMB, endometrial ablation in contrast is less invasive and associated with shorter hospital stay and recovery, significant less pain, and fewer complications [4-7]. Currently, there is a variety of endometrial ablation techniques used worldwide, which are well-established alternatives to hysterectomy for the management of women with HMB $[5,8]$.

Thermachoice endometrial ablation (Gynecare; ethicon Inc., Somerville, NJ, USA) is a non-hysteroscopic ablation technique, involving a heated fluid-filled balloon to thermally ablate the endometrial tissue. This system has undergone significant improvement from the first generation Thermachoice (TBEA) to a third generation Thermachoice III (TBEA-III) in 2003, with the key differences including replacement of the latex balloon with a compliant silicone balloon and introduction of an impeller device for active fluid circulation to enable equal heat distribution to the entire endometrial surface area.

We previously compared the clinical outcome and effectiveness of TBEA and TBEA-III devices and reported 3-years post-treatment follow-up results [9]. The results of that study indicated that the treatment satisfaction was high in both 
cohorts (combined satisfaction rate $76 \%$ ). However, TBEAIII was associated with a higher amenorrhoea rate (35\%) compared to the TBEA device (23\%) [9]. The primary aim of this study was to determine if the treatment success, measured by improvement in bleeding patterns, relief of dysmenorrhoea and patient satisfaction, was maintained over a prolonged period of time i.e., up to 11-year period after the LA-Thermachoice. The secondary aim was to determine if any further medical or surgical procedures such as repeat LAThermachoice, treatment with the LNG-IUS or hysterectomy were required to control symptoms since LA-Thermachoice.

\section{Methods}

This prospective cohort study was carried out in a large teaching hospital. The study population comprised of premenopausal women with heavy menstrual bleeding, refractory to medical treatment and no desire to retain fertility, and who opted for local anaesthetic thermal balloon endometrial ablation (LA-Thermachoice) between February 2001 and December 2011. All patients had preoperative endometrial sampling demonstrating benign histology. These women did not undergo hormonal endometrial thinning in the preoperative preparation. Informed consent for the procedure was obtained by the operating surgeon on the gynaecological ward prior to the procedure.

Women with submucosal fibroids sized $>3 \mathrm{~cm}$, undiagnosed abnormal vaginal bleeding, current lower pelvic infection or uterine abnormalities, atypical endometrial hyperplasia or endometrial cancer, past allergic reactions to local anaesthetic agents, or distorted uterine cavity due to large fibroid, were excluded from the study.

LA-Thermachoice was performed according to our previously described protocol using Thermachoice (Gynecare, Menlo Park, Calif., USA) at study commencement (February 2001-July 2003) and upgraded to Thermachoice III (Gynecare, Ethicon Inc., Somerville, N.J., USA) from August 2003 onwards [10]. The procedure was performed by three consultants over the study period. We maintained a computer database that was populated with the relevant peri-operative information from all patients undergoing LA-Thermachoice since 2001. Data were collected immediately post-procedure and updated once the patient was discharged, with any post procedural complications and analgesia requirements by the operating clinician.

All women were sent a postal questionnaire, including two further postal reminders 3 months apart to non-responders, between February 2012 and August 2012 to evaluate posttreatment symptoms and to determine the long-term effectiveness of therapy (Fig. 1).

Minimum follow-up interval post LA-Thermachoice was 12 months. Symptomatic improvement was assessed by asking women to grade the heaviness of their menstrual blood loss as none (amenorrhea), lighter than before (included spotting and vaginal discharge only), same as before, or heavier than before treatment. They were also asked to quantify their postprocedure menstrual pain, grade their satisfaction with the treatment on a 4-point ordinal scale (very satisfied, satisfied, dissatisfied, or very dissatisfied), and comment on any further
Fig. 1 Flowchart of the study population of patients treated with local anaesthetic thermal balloon endometrial ablation (LA-

Thermachoice) between 2001 and 2011

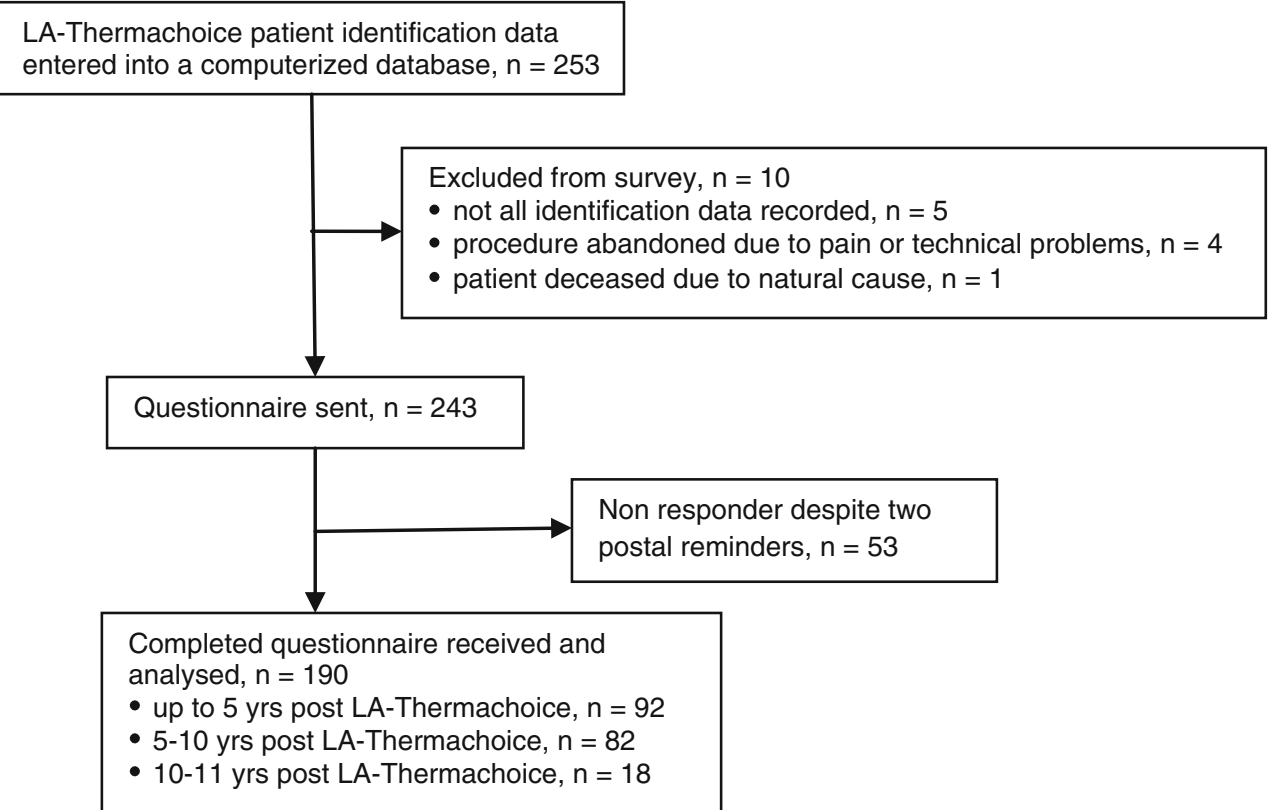


medical or surgical procedures since LA-Thermachoice to control symptoms. All women were advised to quantify their period-related symptoms prior to hysterectomy procedures or menopause if they already had hysterectomy or achieved menopause since LA-Thermachoice. Case notes of non-responders were examined to check if any had undergone hysterectomy since the LA-Thermachoice procedure.

A formal ethics application was not required as the study was classified as a clinical service evaluation. Approval was obtained from the hospital research department to survey all patients who underwent the LA-Thermachoice procedure before the onset of data collection.

Categorical data were summarised as proportions and continuous data as means ( \pm standard deviations (SD)) or medians ( \pm range) where appropriate. Differences between proportions were analysed with the chi-square test with Fisher's exact test. A $p$ value of $<0.05$ was considered as statistically significant. The collected data were analysed using Microsoft Office Excel 2007 and SPSS (version 16, IBM SPSS Statistics).

\section{Findings}

A total of 253 women (mean age 44 years, SD 4.7; BMI 30 \pm 7 ) underwent LA-Thermachoice between February 2001 and December 2011. The procedure was successfully completed in 249 (98\%) women. All baseline clinical characteristics and peri-procedure outcomes of the cohort studied are shown in Table 1.

Fully completed questionnaires were returned by 190 participants $(78 \%)$. One woman was deceased at the time of the first mailing. Median follow-up was 71 months (Range 8-134) (Table 2).

Seventy-nine percent of responders reported significant improvement in HMB and majority felt that the benefit of the procedure was maintained over a long period of time (Table 2). Ten (5\%) responders reported that they had undergone menopause since the LA-Thermachoice and of these, six (3\%) were using hormone replacement therapy (HRT). Twenty $(11 \%)$ responders reported that they had a hysterectomy. Both the above groups were excluded from the analysis for improvement in menstrual symptoms. Overall, 161 (86 \%) reported that they were satisfied or very satisfied with the outcome and would recommend the procedure to a friend or a family member. The high satisfaction with endometrial ablation which was noticed in our previous study [9] was maintained for over an 11-years period of time (Table 2) since the procedure.

The satisfaction rates with the procedure in responders with up to 6 years follow-up $(n=96)$ were similar to those in
Table 1 Demographics and intraoperative data of women undergoing local anaesthetic thermal balloon endometrial ablation (LAThermachoice) between 2001 and 2011, $n=253$

\begin{tabular}{|c|c|}
\hline Variable & Number $(\%)$ \\
\hline \multicolumn{2}{|l|}{ Age } \\
\hline Mean & 44 \\
\hline SD & 4.7 \\
\hline \multicolumn{2}{|l|}{ BMI, $n=240$} \\
\hline Mean & 30 \\
\hline SD & 6.8 \\
\hline \multicolumn{2}{|l|}{ Cycle phase, $n=247$} \\
\hline Proliferative & $93(37 \%)$ \\
\hline Secretary & $91(37 \%)$ \\
\hline Menstruating & $34(14 \%)$ \\
\hline Mid cycle & $15(6 \%)$ \\
\hline Amenorrhoea due to drugs & $14(6 \%)$ \\
\hline \multicolumn{2}{|c|}{ Clinical findings (USS or hysteroscopic), $n=249$} \\
\hline Normal & $191(77 \%)$ \\
\hline Intramural fibroid & $39(15 \%)$ \\
\hline Submucosal fibroid $<3 \mathrm{~cm}$ & $5(2 \%)$ \\
\hline Polyp & $7(3 \%)$ \\
\hline Other & $7(3 \%)$ \\
\hline \multicolumn{2}{|l|}{ Uterine size, $\mathrm{cm}, n=249$} \\
\hline$<10$ & $184(74 \%)$ \\
\hline$\geq 10$ & $43(17 \%)$ \\
\hline Not recorded & $22(9 \%)$ \\
\hline Mean size & 8.6 \\
\hline SD & 1.2 \\
\hline \multicolumn{2}{|l|}{ Volume of fluid, ml } \\
\hline \multicolumn{2}{|l|}{ Thermachoice, $n=51$} \\
\hline Mean & 24.1 \\
\hline $\mathrm{SD}$ & 13.3 \\
\hline \multicolumn{2}{|l|}{ Thermachoice III, $n=185$} \\
\hline Mean & 19.1 \\
\hline $\mathrm{SD}$ & 11.2 \\
\hline \multicolumn{2}{|l|}{ Intrauterine pressure, $\mathrm{mm} \mathrm{Hg}$} \\
\hline \multicolumn{2}{|l|}{ Thermachoice, $n=51$} \\
\hline Mean & 157 \\
\hline SD & 16 \\
\hline \multicolumn{2}{|l|}{ Thermachoice III, $n=188$} \\
\hline Mean & 179 \\
\hline SD & 16 \\
\hline \multicolumn{2}{|c|}{ Mean visual analogue pain (VAS) immediately after procedure, $n=234$} \\
\hline Mean & 5.5 \\
\hline SD & 2.7 \\
\hline Not severe (VAS $\leq 8)$ & $200(81 \%)$ \\
\hline Severe $($ VAS $\geq 9$ ) & $34(14 \%)$ \\
\hline
\end{tabular}

Data not reported in all cases; calculations were based on cases that were recorded 
Table 2 Questionnaire survey depicting long-term outcomes in women undergoing local anaesthetic thermal balloon endometrial ablation (LAThermachoice) between 2001 and 2011

\begin{tabular}{|c|c|c|}
\hline \multirow[t]{2}{*}{ Variable } & \multicolumn{2}{|l|}{ Varma et al. [9] } \\
\hline & $\begin{array}{l}\text { 2001-2005 } \\
\text { Up to 3-year f/up }\end{array}$ & $\begin{array}{l}\text { 2001-2011 } \\
\text { Up to 11-year f/up }\end{array}$ \\
\hline Response rate & $n=88(86 \%)$ & $n=190(78 \%)^{\mathrm{a}}$ \\
\hline Mean follow-up time, months & 30 & 62 \\
\hline Range & $12-54$ & $12-134$ \\
\hline $\mathrm{SD}$ & 13 & 42 \\
\hline \multicolumn{3}{|l|}{ Periods at review } \\
\hline Amenorrhoea & $27(29 \%)$ & $106(56 \%)^{\mathrm{b}}$ \\
\hline Lighter & $51(55 \%)$ & $75(39 \%)$ \\
\hline No change or worse & $15(16 \%)$ & $9(5 \%)$ \\
\hline \multicolumn{3}{|c|}{ Length of bleeding at review, $n=188$} \\
\hline No bleeding & & $106(56 \%)$ \\
\hline$<3$ days & & $26(14 \%)$ \\
\hline $3-5$ days & & $27(14 \%)$ \\
\hline 6-7 days & & $16(9 \%)$ \\
\hline 8-10 days & & $6(3 \%)$ \\
\hline$>10$ days & & $7(4 \%)$ \\
\hline \multicolumn{3}{|l|}{ Dysmenorrhoea at review } \\
\hline Less pain or no pain & $64(69 \%)$ & $167(86 \%)$ \\
\hline No change & $11(12 \%)$ & $18(9 \%)$ \\
\hline Worsening & $18(19 \%)$ & $10(5 \%)$ \\
\hline \multicolumn{3}{|c|}{ Benefit maintained over time, $n=185$} \\
\hline Yes & & $149(81 \%)$ \\
\hline No & & $36(19 \%)$ \\
\hline \multicolumn{3}{|c|}{ Further treatment (repeat ablation, drugs, LNG-IUS or hysterectomy), $n=187$} \\
\hline No & $83(81 \%)$ & $166(89 \%)$ \\
\hline Yes & $19(19 \%)$ & $21(11 \%)$ \\
\hline \multicolumn{3}{|c|}{ Reason for further treatment, $n=21$} \\
\hline Bleeding & & $6(29 \%)$ \\
\hline Pain & & $8(38 \%)$ \\
\hline Both & & $7(33 \%)$ \\
\hline \multicolumn{3}{|l|}{ All types of further treatment } \\
\hline No further treatment & $74(73 \%)$ & $156(82 \%)$ \\
\hline LNG-IUS & $3(3 \%)$ & $4(2 \%)$ \\
\hline Drugs (including HRT) & $9(9 \%)$ & $9(5 \%)$ \\
\hline Repeat endometrial ablation & $2(2 \%)$ & $1(0 \%)$ \\
\hline Hysterectomy $^{\mathrm{c}}$ & $14(14 \%)$ & $20(11 \%)$ \\
\hline \multicolumn{3}{|l|}{ Satisfaction, $n=186$} \\
\hline Very satisfied or satisfied & $78(76 \%)$ & $161(86 \%)$ \\
\hline Dissatisfied & $24(24 \%)$ & $25(14 \%)$ \\
\hline
\end{tabular}

$L N G$-IUS = levonorgestrel releasing intrauterine system

${ }^{a}$ Data not recorded in all cases; calculations were based on cases that were reported

${ }^{\mathrm{b}}$ Ten $(5 \%)$ responders reported that they had undergone menopause since the LA-TBEA

${ }^{\mathrm{c}}$ Histology of the 16 hysterectomies reported adenomyosis, leiomyoma, both adenomyosis and leiomyoma, no histological pathology, and no data recorded in $6,2,3,5$, and 4 cases,

respectively responders between 6 and up to 11 years $(n=90)$ follow-up (79 vs. $84 \%, p>0.05$ ).

Secondary treatment with the LNG-IUS (2\%), repeat TBEA $(0.5 \%)$, or hysterectomy (11\%) occurred in 34/190 (18\%) responders. Of the $20(11 \%)$ responders who indicated they had a hysterectomy following the LA-Thermachoice, 6 reported they were still satisfied with the initial procedure. These patients reported the main reason for the hysterectomy as dysmenorrhoea except for one responder who underwent a hysterectomy 10 years following the LA-Thermachoice due to a possible endometrial cancer.

In order to ensure a complete long-term follow-up, we examined the case notes of all non-responder patients for an entry of a hysterectomy operation over this period. In 15 patients, we cannot be sure if any additional surgery has been carried out elsewhere. Eighteen out of 53 (34\%) nonresponders had a hysterectomy. Histology of these 18 hysterectomies reported adenomyosis $(n=3)$, leiomyoma $(n=2)$, adenomyosis with leiomyoma $(n=4)$, and no histological abnormality $(n=9)$. The remaining $(n=20)$ patients have not had any surgical interventions since their LA-Thermachoice.

Therefore, in total, only 38 out of $243(16 \%)$ patients required hysterectomy post LA-Thermachoice to control their symptoms. The average time interval between LAThermachoice procedure and hysterectomy was 22 months (SD 17). Hysterectomy rate was higher in patients who underwent endometrial ablation with TBEA $(n=15 / 51$ i.e., $29 \%$ users) compared to TBEA-III ( $n=23 / 192$ i.e., $12 \%$ users) device.

\section{Discussion}

This study represents the largest published series of local anaesthetic endometrial ablation. To the best of our knowledge, this is the first study to report clinical outcomes with 11 years (median 71 months) of post LA-Thermachoice follow-up. The study results show that the local anaesthetic outpatient thermal balloon endometrial ablation is an effective minimally invasive procedure for the management of women with symptoms of HMB. The results confirm that the patient satisfaction with LA-Thermachoice is high, and is maintained over a long period of time after the procedure. This study is important in that it provides further data on the long-term outcome and success of LA-Thermachoice.

Review of the literature data evaluating patient satisfaction and clinical outcomes with the LA-Thermachoice reveal a wide variation in results. Many of the studies are small and have a shorter follow-up time [11, 12]. It is important to follow the patients for longer periods, as some outcomes may change over time.

The main strength of this study lies in its long-term followup as we were able to cover a significant post-procedure 
follow-up time frame up to 134 months (median 71 months). The reported satisfaction rates with LA-Thermachoice in our study are in agreement with previously reported satisfaction rates (range 80-95\%) from women who underwent TBEA for HMB, proving the validity of the questionnaire survey [13-18].

There may be limitations in this study which lessens the reliability of its conclusions. The study population may be heterogeneous in relation to different heavy menstrual bleeding patterns. The study did not collect baseline quality-of-life data but we used established methods of assessing symptoms compared to pre-treatment. Also, it is possible that the $5 \%$ of women who have subsequently undergone menopause would positively bias the results, but we were careful that we discounted these patients from the results which still showed significant improvement in patient outcomes. This could be particularly so as patients who underwent treatment from the TBEA device (older population of follow-up), which was shown to have a poorer outcome compared to TBEA-III device, have subsequently undergone the menopause [9]. All women were advised to quantify their period-related symptoms prior to menopause if they already had achieved menopause since LA-Thermachoice. To minimise this bias, we have reported our figures after excluding those women who had undergone the menopause. This therefore represents the most conservative but pragmatic situation regarding the longterm outcome following LA-Thermachoice. Another important issue for questionnaire research is responder and non-responder bias. To minimise this bias, we tried to increase our response rate by contacting eligible patients multiple times. We managed to receive a good response rate to our survey, with $78 \%$ of women completing the survey, which was better than the mean response of other mail surveys [19]. The response rate may reflect women who are not willing to respond to postal questionnaires, those who may not recall their symptoms before LAThermachoice, natural loss of patients in long-term studies, and those where questionnaire was ultimately determined undeliverable due to incorrect address.

\section{Conclusion}

The study data confirms that patient satisfaction with LAThermachoice is high, and is maintained over a long period of time after the procedure. Furthermore, the data confirms that LA-Thermachoice is an effective treatment option for women with symptoms of $\mathrm{HMB}$ and a robust alternative to hysterectomy. This information is valuable when counselling patients regarding long-term satisfaction, success in reducing HMB and dysmenorrhoea with LA-Thermachoice, and potential need for further surgery after LA-Thermachoice.

\section{References}

1. Sculpher MJ, Bryan S, Dwyer N, Hutton J, Stirrat GM (1993) An economic evaluation of transcervical endometrial resection versus abdominal hysterectomy for the treatment of menorrhagia. $\mathrm{Br} \mathrm{J}$ Obstet Gynaecol 100:244-252

2. Shapley M, Jordan K, Croft PR (2004) An epidemiological survey of symptoms of menstrual loss in the community. Br J Gen Pract 54:359-363

3. Gupta J, Kai J, Middleton L, Pattison H, Gray R, Daniels J (2013) Levonorgestrel intrauterine system versus medical therapy for menorrhagia. N Engl J Med 368:128-137

4. Cooper K, Lee A, Chien P, Raja E, Timmaraju V, Bhattacharya S (2011) Outcomes following hysterectomy or endometrial ablation for heavy menstrual bleeding: retrospective analysis of hospital episode statistics in Scotland. BJOG 118:1171-1179

5. Pados G, Athanatos D, Tsolakidis D, Stamatopoulos P, Tarlatzis B (2011) Treatment options for dysfunctional uterine bleeding: evaluation of clinical results. GynecolSurg 8:385-393

6. Middleton LJ, Champaneria R, Daniels JP et al (2010) Hysterectomy, endometrial destruction, and levonorgestrel releasing intrauterine system (Mirena) for heavy menstrual bleeding: systematic review and meta-analysis of data from individual patients. BMJ 341:c3929

7. Clark TJ, Samuels N, Malick S, Middleton L, Daniels J, Gupta J (2011) Bipolar radiofrequency compared with thermal balloon endometrial ablation in the office: a randomized controlled trial. Obstet Gynecol 117:1228

8. Matteson KA, Abed H, Wheeler TL, Sung VW, Rahn DD, Schaffer JI, Balk EM (2012) A systematic review comparing hysterectomy with less-invasive treatments for abnormal uterine bleeding. J Minim Invasive Gynecol 19:13-28

9. Varma R, Soneja H, Samuel N, Sangha E, Clark TJ, Gupta JK (2010) Outpatient Thermachoice endometrial balloon ablation: long-term, prognostic and quality-of-life measures. Gynecol Obstet Invest 70:145-148

10. Clark TJ, Gupta JK (2004) Outpatient thermal balloon ablation of the endometrium. Fertil Steril 82:1395-1401

11. Jarrell A, Olsen ME (2003) Patient satisfaction with thermal balloon endometrial ablation. A retrospective review. J Reprod Med 48:635636

12. Vilos GA, Fortin CA, Sanders B, Pendley L, Stabinsky SA (1997) Clinical trial of the uterine thermal balloon for treatment of menorrhagia. J Am Assoc Gynecol Laparosc 4:559-565

13. Cash C Jr, Garza-Leal J, Donovan A et al (2012) Clinical evaluation of long-term safety and effectiveness of a third-generation thermal uterine balloon therapy system for heavy menstrual bleeding. J Minim Invasive Gynecol 19:469-476

14. Chapa HO, Antonetti AG, Venegas G, Sandate J, VanDuyne CP (2010) Menstrual and dysmenorrhea outcomes after ThermaChoice III: a 2-year prospective study. J Gynecol Surg 25:233-236

15. Cooley S, Yuddandi V, Walsh T, Geary M, McKenna P (2005) The medium- and long-term outcome of endometrial ablative techniques. Eur J Obstet Gynecol Reprod Biol 121(2):233-5

16. Hazard D, Harkins G (2009) Patient satisfaction with thermal balloon ablation for treatment of menorrhagia. Am J Obstet Gynecol 200:e21-e23

17. Andersson S, Mints M (2007) Thermal balloon ablation for the treatment of menorrhagia in an outpatient setting. Acta Obstet Gynecol Scand 86:480-483

18. Kleijn JH, Engels R, Bourdrez P, Mol BW, Bongers MY (2008) Fiveyear follow up of a randomised controlled trial comparing NovaSure and ThermaChoice endometrial ablation. BJOG 115:193-198

19. Asch DA, Jedrziewski MK, Christakis NA (1997) Response rates to mail surveys published in medical journals. J Clin Epidemiol 50:1129-1136 